\title{
Development of a Photoactivatable Phosphine Probe for Induction of Intracellular Reductive Stress with Single-Cell Precision
}

\author{
Journal Article \\ Author(s): \\ Tirla, Alina; Rivera-Fuentes, Pablo \\ Publication date: \\ 2016-11-14 \\ Permanent link: \\ https://doi.org/10.3929/ethz-b-000123593 \\ Rights / license: \\ In Copyright - Non-Commercial Use Permitted \\ Originally published in: \\ Angewandte Chemie. International Edition 55(47), https://doi.org/10.1002/anie.201608779
}




\title{
Development of a Photoactivatable Phosphine Probe for Induction of Intracellular Reductive Stress with Single-Cell Precision
}

\author{
Alina Tirla and Pablo Rivera-Fuentes* \\ Laboratorium für Organische Chemie, ETH Zürich, \\ HCl G329, Vladimir-Prelog-Weg 3, 8093 Zürich, Switzerland. \\ E-mail: pablo.rivera-fuentes@org.chem.ethz.ch
}

\begin{abstract}
Photoactivatable phosphines that induce intracellular reductive stress are reported. The design of these probes takes advantage of the conjugate addition of trialkylphosphines to carbocyanine dyes, which can be reverted photochemically to produce the trialkylphosphine and a fluorescent reporter. The photochemical release depends on the efficiency of photoinduced electron transfer from the indolenine arm of the probe to the coumarin acceptor. These probes permeate readily the mammalian plasma membrane and can be photoactivated in live cells. Upon irradiation of the probe, the released trialkylphosphine induces intracellular reductive stress, which ultimately leads to formation of Thioflavin-positive intracellular protein aggregates. These effects could be induced in individual cells within a monolayer, with minimal disturbance of neighboring cells.
\end{abstract}

Concentrations of reducing and oxidizing agents are tightly controlled in biological systems (redox homeostasis).$^{[1]}$ This balance is obtained by regulating the ratios of redox pairs, mainly glutathione (GSH) and its oxidized, disulfide-bonded form (GSSG). ${ }^{[2]}$ Disruption of redox homeostasis that produces excessively reducing environments has been associated with numerous pathologies, including cancer, inflammation, metabolic disorders, and neurodegenerative diseases. ${ }^{[3]}$ Investigation of the molecular mechanisms of reductive stress will contribute to our understanding of redox homeostasis and ultimately inform the design of improved therapies for diseases associated to this crucial aspect of cell biology.

In the laboratory, reductive stress can be induced employing pharmacological agents, in particular thiols such as $\mathrm{N}$-acetyl-L-cysteine ${ }^{[4]}$ or dithiothreitol (DTT). ${ }^{[5]}$ These reducing agents are efficient but lack spatial resolution, for instance, they do not distinguish between individual cells within a multicellular environment. Considering that different cell types within tissue might display different responses to disruption of redox homeostasis, it would be advantageous to be able to induce reductive stress using a reagent that acts rapidly, on demand and with single-cell precision. Photoactivatable (often called "caged" or "photocaged") probes fulfill these requirements by providing spatially and temporally controlled release of bioactive agents. ${ }^{[6,7]}$ The utility of these molecular tools has been thoroughly demonstrated and many photoactivatable ions, ${ }^{[9]}$ neurotransmitters, ${ }^{[10]}$ lipids, ${ }^{[11]}$ nucleic acids, ${ }^{[12]}$ peptides, ${ }^{[13]}$ and proteins ${ }^{[14]}$ have been developed. Herein, we report the design, synthesis and implementation of a photoactivatable phosphine, which permeates the plasma membrane and, upon photoactivation, release a reporter fluorescent dye and a trialkylphosphine that induces reductive stress in live cells.

Trialkylphosphines reduce biological disulfide bonds efficiently. ${ }^{[15]}$ We envisioned that a photoactivatable trialkylphosphine would be an effective inducer of intracellular reductive stress. Very recently, the first photoactivatable triarylphosphines were reported in the context of light-activated, Bertozzi-Staundiger ligations. ${ }^{[16,17]}$ Despite the value of these probes, we were interested in developing a photoactivatable group that, upon irradiation with visible light, would produce a strongly reducing trialkylphosphine and a fluorescent reporter as the only two photoproducts. With these requirements in mind, we based our design on the conjugate addition reaction of tris(2-carboxyethyl)phosphine (TCEP) to carbocyanine dye Cy5, which can be reversed upon irradiation with UV light (Scheme 1). ${ }^{[18]}$ The carbocyanine-phosphonium adduct, however, is in fast equilibrium with the dye and free phosphine at room temperature and is not an isolable species. ${ }^{[18]}$

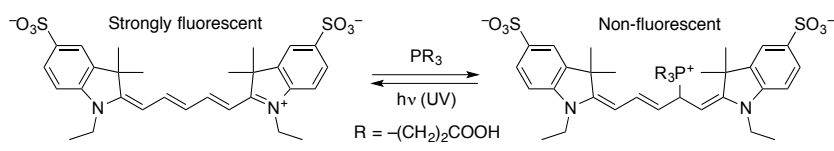

Scheme 1. Conjugate addition of tris(2-carboxyethyl)phosphine (TCEP, $\mathrm{PR}_{3}$ ) to carbocyanine Cy5 and photochemical elimination of the phosphine. ${ }^{[18]}$ 
To develop a carbocyanine-phosphonium adduct with improved thermal stability, density functional theory (DFT) calculations were performed to estimate the barrier of phosphine dissociation in the electronic ground state (for details see the Supporting Information -SI-). Starting with the carbocyanine Cy5 adduct, the substituents on the cyanine backbone were varied systematically to increase the thermal (non-photochemical) barrier of $\mathrm{C}-\mathrm{P}$ bond cleavage. These modeling revealed that desymmetrization of the cyanine dye and introduction of weak electron-donor substituents ( $N, N$-dimethylaminocoumarin) and electron-withdrawing indolenines increased the predicted stability of the phosphonium adduct. Based on these calculations, Compounds 1a-d (Figure 1) were selected as models for the synthesis of a series of probes.
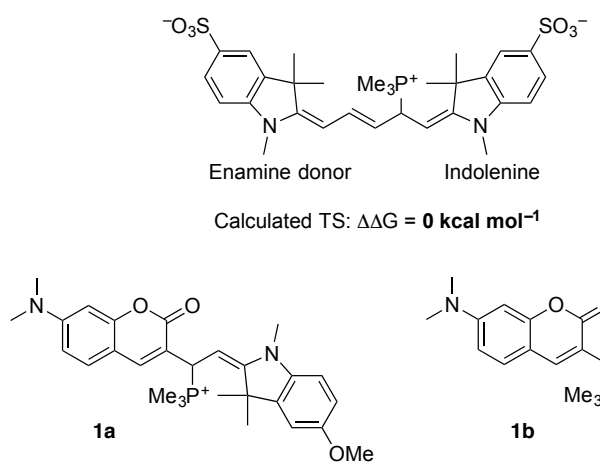
$N, N$-dimethylaminocoumarin Indolenine
donor
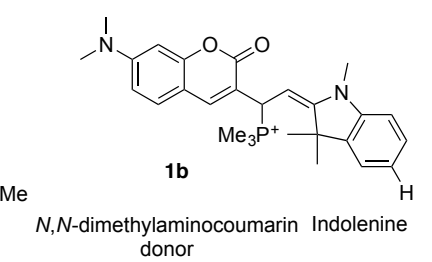

Calculated TS: $\Delta \Delta \mathrm{G}=2.7 \mathrm{kcal} \mathrm{mol}^{-1}$ donor

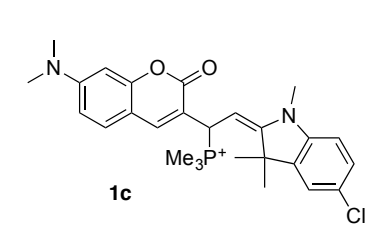

$\mathrm{N}, \mathrm{N}$-dimethylaminocoumarin Indolenine donor

Calculated TS: $\Delta \Delta G=\mathbf{3 . 3} \mathrm{kcal} \mathrm{mol}^{-1}$

Calculated TS: $\Delta \Delta G=3.4 \mathrm{kcal} \mathrm{mol}^{-1}$

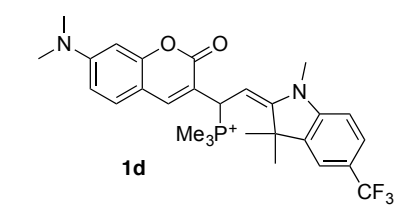

$\mathrm{N}, \mathrm{N}$-dimethylaminocoumarin Indolenine donor

Calculated TS: $\Delta \Delta \mathrm{G}=\mathbf{3 . 8} \mathrm{kcal} \mathrm{mol}^{-1}$

Figure 1. Computational design of carbocyanine-phosphonium adducts with increased stability compared to that of Cy5 (top). All transition-state energies are provided relative to that of the Cy5 adduct $\left(0 \mathrm{kcal} \mathrm{mol}^{-1}\right)$.

The synthesis of photoactivatable phosphines started with the condensation of Fischer's bases 2a-d with aldehyde 3 to obtain the respective coumarin-carbocyanine dyes 4 a-d. ${ }^{[19]}$ These dyes were treated with $n-$ tributylphosphine to give phosphonium compounds 5a-d (Scheme 2), which, unlike Cy5 adducts, could be isolated as pure compounds. ${ }^{1} \mathrm{H}-{ }^{31} \mathrm{P}$ HMBC NMR experiments were carried out to confirm the regioselectivity of the conjugate addition (Figure S1).

Compounds $\mathbf{5 a - d}$ and $\mathbf{4 a - d}$ are soluble in phosphate-buffered saline (PBS) up to $\sim 400 \mu \mathrm{M}$ concentration. All absorbance and fluorescence spectra were measured in PBS (2 $\mu \mathrm{M}$, Figures S2-3). Compounds 4a-d display strong absorbance around $570 \mathrm{~nm}$, whereas compounds 5a-d have absorption maxima around 415 $\mathrm{nm}$. Absorption and NMR spectroscopic analyses suggested that compounds 5a-d decomposed in solution at various rates to produce dyes $\mathbf{4 a - d}$ and $n$-tributylphosphine (Scheme $2 \mathrm{~B}$ ), which was characterized by decreased absorption at 415 and increase around $570 \mathrm{~nm}$. The rates of phosphine release for compounds $5 a-$ d were determined in the absence of photoactivating light to evaluate their inherent thermal stability (Figure S4 and Table S1), but in the presence of excess GSSG, which reacts readily with $n$-tributylphosphine (Figure S5), making the reaction irreversible. Compounds $\mathbf{5 a - d}$ displayed markedly different rates of phosphine release in the absence of light (Figure S4 and Table S1). The stability of the carbocyanine-phosphonium adduct increased as a function of the electron-withdrawing ability of the substituent on the indolenine, confirming the trend obtained computationally (Figure 1). To evaluate the ability of compounds 5a-d to release phosphine upon photoactivation, a solution of $\mathbf{5 b}$ was irradiated under aerobic conditions using an LED source (405 $\mathrm{nm}$, $10 \mathrm{~mW}) .{ }^{1} \mathrm{H},{ }^{31} \mathrm{P}$ NMR and absorbance spectra confirmed that the only two photoproducts were dye $\mathbf{4 b}$ and $n$ tributylphosphine oxide (Figure S6). 

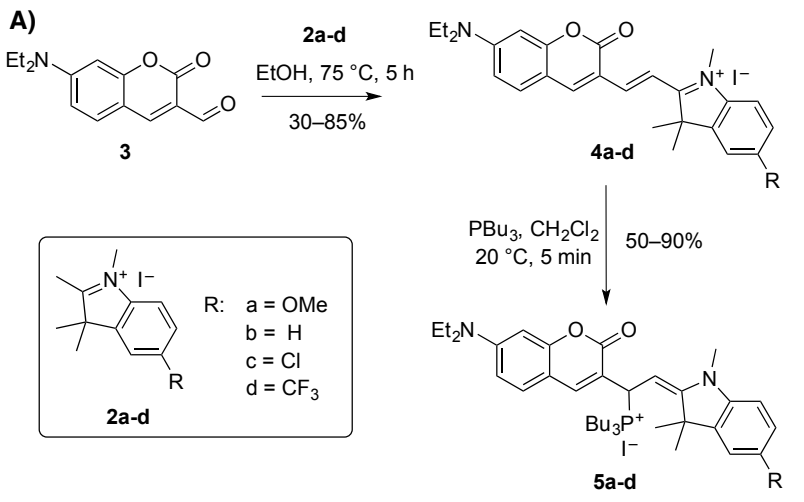

B)

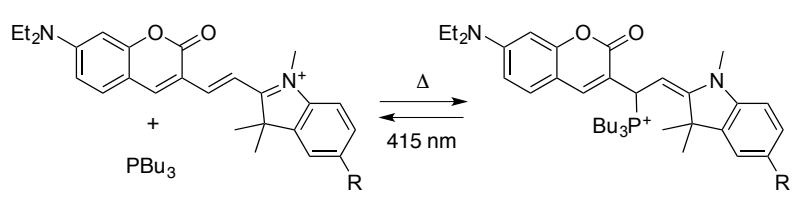

Scheme 2. A) Synthesis of probes 5 a-d, B) Thermal formation of the phosphonium adduct and photochemical release of $n$ tributylphosphine and dyes $4 a-d$.

Photoactivation experiments revealed that compounds 5a-c release $n$-tributylphosphine and dyes 4 a-c, albeit with low photoactivation quantum yields (Tables S1 and S2). In contrast, probe $\mathbf{5 d}$ did not react under irradiation. This lack of reactivity revealed a possible mechanism of photoactivation. A combination of theoretical calculations and photophysical experiments (Figures S7-10 and Scheme S1) revealed that for compounds $\mathbf{5 a - c}$, but not $\mathbf{5 d}$, excitation of the coumarin fragment leads to photoinduced electron transfer $(\mathrm{PeT})$ from the indolenine arm. This further enhancement of the electron-donating ability of the coumarin triggers the elimination of phosphine in a manner akin to that of self-immolative linkers. ${ }^{[20]}$

Based on their thermal stabilities and photochemical cleavage, probes $\mathbf{5 b}$ and $\mathbf{5 c}$ were subsequently tested in live cells. Results for $\mathbf{5 c}$ are shown here and those for $\mathbf{5 b}$ are presented in the SI. Human cervical cancer cells (HeLa) were treated with $\mathbf{5 c}$, washed, and the fluorescence of the cells was recorded in the red channel before and after exposure to the $405 \mathrm{~nm}$ laser of the microscope (Figure 2A-C). This experiment demonstrated that probe $\mathbf{5 c}$ was present in the intracellular medium and that the compound can be efficiently photoactivated (for a kinetic trace of live-cell photoactivation, see Figure S11). Moreover, when the cells were not irradiated, only negligible increase in red fluorescence was recorded after $>60$ min (Figure S12). In addition, probes $\mathbf{5 b}$ and $\mathbf{5 c}$ did not seem to reduce the viability of HeLa cells at the concentrations and incubation times used for imaging experiments (see the $\mathrm{SI}$ ).

Photoactivation of probe $\mathbf{5 c}$ efficiently converted GSSG to GSH in vitro (Figure S5), demonstrating that it can produce free thiols from disulfides. To test whether $5 \mathrm{c}$ could also increase the concentration of free thiols in live cells, monobromobimane (mBB) was employed, which is a non-specific, thiol-sensitive, fluorescent probe. ${ }^{[21]}$ HeLa cells were treated with $\mathrm{mBB}$ and $\mathbf{5 c}$, and exposed to $405 \mathrm{~nm}$ light. These cells displayed a 6fold increase in $\mathrm{mBB}$ fluorescence compared to a control sample that was treated with $\mathrm{mBB}$ and irradiated in the absence of 5c (Figure 2D-F). Notably, mBB could undergo reductive dehalogenation upon direct reaction with a trialkylphosphine, which would produce a fluorescent product (Figure S13). ${ }^{[2]}$ Although we cannot rule out this possibility, this experiment showed that species capable of reducing mBB were formed upon photoactivation of $\mathbf{5 c}$ in live cells.

Cleavage of disulfide bonds in proteins, leading to unfolding or misfolding and aggregation, is a consequence of reductive stress. ${ }^{[23]}$ To test whether intracellular protein aggregation is triggered by photoactivation of $\mathbf{5 c}$, imaging experiments were carried out employing Thioflavin T (ThT), a common stain for protein amyloid aggregates. ${ }^{[24]}$ Live HeLa cells treated with 5c and ThT displayed a 13-fold stronger ThT emission after photoactivation compared to control cells that were irradiated and stained with ThT, but not treated with $5 \mathrm{c}$ (Figure $2 \mathrm{G}-\mathrm{I}$ ). 


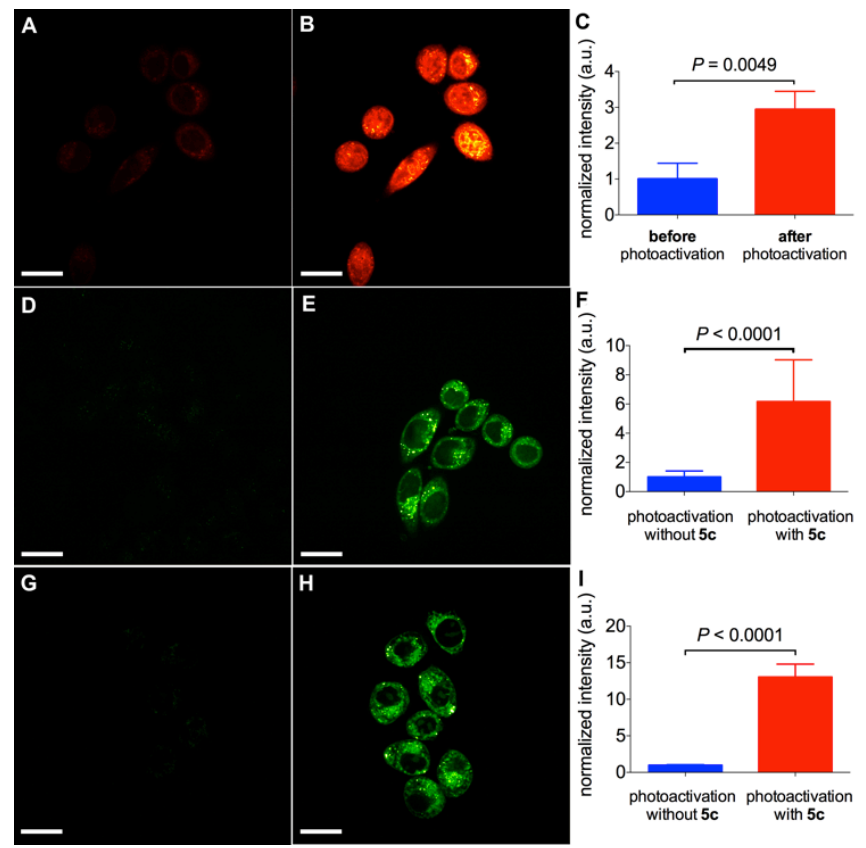

Figure 2. Photoactivation of compound $\mathbf{5 c}$ in live HeLa cells. A) Cells after 10 min incubation with $\mathbf{5 c}(10 \mu \mathrm{M})$ before photoactivation. B) Same cells as in (A) after photoactivation ( $405 \mathrm{~nm}, 120 \mathrm{~mW}, 20 \%$ power, $25 \mathrm{~s}$ ). C) Quantification of the fluorescence intensity of 5c (details of data collection and statistical analysis are described in the SI). D) Cells treated only with mBB (50 $\mu \mathrm{M})$ after photoactivation E) Generation of intracellular reducing agents in cells treated with $\mathrm{mBB}(50 \mu \mathrm{M})$ and $\mathbf{5 c}(10 \mu \mathrm{M})$ after photoactivation. F) Quantification of the fluorescence intensity of $\mathrm{mBB}$. G) Cells treated with ThT $(5 \mu \mathrm{M})$ and photoactivated. H) Generation of intracellular protein aggregates in cells treated with $\mathbf{5 c}(10 \mu \mathrm{M})$ for $10 \mathrm{~min}$, then photoactivated, washed and stained with ThT $(5 \mu \mathrm{M})$. l) Quantification of fluorescence intensity of ThT. $\mathrm{mBB}=$ monobromobimane, ThT $=$ Thioflavin T. Scale bar $=10 \mu \mathrm{m}$.

Moreover, irradiation of a solution of ThT, either pure or in the presence of excess $\mathbf{5 c}$, did not increase the fluorescence of the former significantly (Figure S14-15). These experiments suggest that photoactivation of 5c induces the formation of intracellular protein aggregates that can be stained with ThT.

Photoactivatable probes are particularly useful for experiments that require stimulation of an individual cell within a complex population, for example, to investigate cellular connectivity. ${ }^{[25]}$ We used our probes to perform photoactivation experiments in individual cells within a monolayer. Cells were incubated with $\mathbf{5 b}$ or $\mathbf{5 c}$, washed and imaged in the red channel. A region of interest (ROI) comprising a single cell was selected and irradiated with a $405 \mathrm{~nm}$ laser pulse $\left(120 \mathrm{~mW}, 50 \%\right.$ power, $\left.0.5 \mathrm{~ms}_{\text {pixel }}{ }^{-1}\right)$, similar to a fluorescence recovery after photobleaching (FRAP) experiment. ${ }^{[26]}$ Figure 3 shows the results obtained with probe $\mathbf{5 c}$ (results for $\mathbf{5 b}$ can be found in the SI). Immediately after the FRAP pulse, the intracellular fluorescence intensity increased $\sim 10$-fold (Figure 3C). A time-lapse experiment (Movie S1) revealed that the fluorescence intensity of 5c decreases steadily after photoactivation (Figure S21) owing to either photobleaching of the dye or diffusion of the probe to the extracellular space. The combination of these effects may account for the smaller increase observed in experiments with larger photoactivated areas and longer exposure times (Figure 2). 

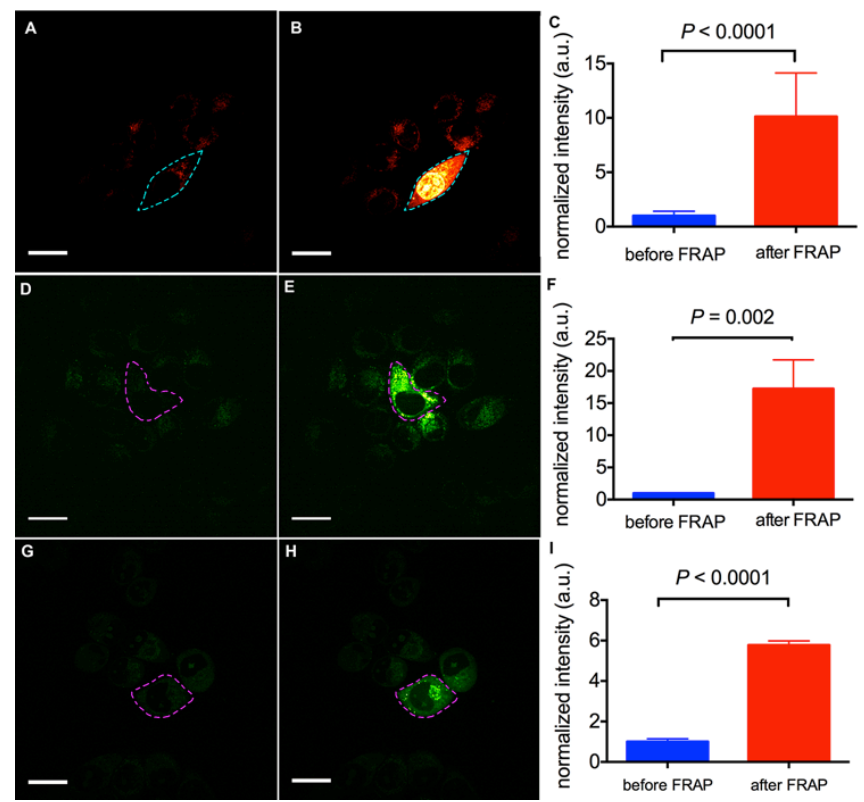

Figure 3. Single-cell photoactivation of $\mathbf{5 c}$. A) Cells treated with $\mathbf{5 c}(10 \mu \mathrm{M})$ before photoactivation. B) Same cells as in (A) after photoactivation by a FRAP pulse $\left(405 \mathrm{~nm}, 120 \mathrm{~mW}, 50 \%\right.$ power, $\left.0.5 \mathrm{~ms}^{\text {pixel }}{ }^{-1}\right)$ in a ROI (dashed cyan line). C) Quantification of fluorescence intensity of $\mathbf{5 c}$ in the ROI. D) Cells treated with $\mathrm{mBB}(50 \mu \mathrm{M})$ and $\mathbf{5 c}(10 \mu \mathrm{M})$ before photoactivation. E) Same cells as in (D) after photoactivation by a FRAP pulse $\left(405 \mathrm{~nm}, 120 \mathrm{~mW}, 50 \%\right.$ power, $0.5 \mathrm{~ms}^{\text {pixel }} \mathrm{l}^{-1}$ ) in a ROI (dashed magenta line). $\mathrm{F}$ ) Quantification of the fluorescence intensity of mBB in the ROI. G) Cells treated with ThT $(5 \mu \mathrm{M})$ and $\mathbf{5 c}(10 \mu \mathrm{M})$ before photoactivation $\mathrm{H})$ Same cells as in (G) after photoactivation by a FRAP pulse $\left(405 \mathrm{~nm}, 120 \mathrm{~mW}, 50 \%\right.$ power, $\left.0.5 \mathrm{~ms}^{\text {pixel }}{ }^{-1}\right)$ in a ROI (dashed magenta line). I) Quantification of the fluorescence intensity of ThT in the ROI. ROI = region of interest, Scale bar $=10 \mu \mathrm{m}$.

Photoactivation of $\mathbf{5 c}$ in individual cells led to production of reducing species detectable by $\mathrm{mBB}$ under the staining conditions used before (Figure 3D-F). Similarly, photoactivation of $\mathbf{5 c}$ in a single cell induced the formation of protein aggregates that could be detected by ThT staining following the procedure described above (Figure 3G-I). In both cases, irradiation of cells in the absence of probe 5c did not produce any significant increase in fluorescence of either mBB or ThT (Figures S24-25).

In summary, a series of fluorogenic, photoactivatable phosphine probes was developed. A combination of computational modeling, synthesis, and mechanistic studies yielded probes that are suitable for live cell photoactivation experiments. These molecules permeate the intact membrane of live mammalian cells and can be activated with visible light to increase the intracellular concentration of reducing species and form protein aggregates, both of which are hallmarks of intracellular reductive stress. By employing these probes, we were able to induce reductive stress in individual cells with minimal perturbation of neighboring cells in a monolayer. This experiment highlights the unprecedented spatiotemporal resolution that can be achieved with these photoactivatable compounds. Current work in our laboratory is aimed at improving this spatial resolution further to be able to induce reductive stress in specific subcellular compartments.

\section{Acknowledgements}

This work was supported by ETH Zurich and the Swiss National Science Foundation (grant 200021_165551). Confocal microscopy was carried out at the Scientific Center for Optical and Electron Microscopy (ScopeM) at ETH Zurich and computational work was performed on the Euler cluster of the Swiss National Supercomputing Center. We thank Dr. Ferry Prins (ETH Zurich) for carrying out excited-state lifetime measurements and all research groups at the Laboratory of Organic Chemistry (ETH Zurich) for access to instruments and continuous support.

Keywords: fluorescent probes $\bullet$ imaging agents $\bullet$ photoactivation $\bullet$ reduction $\bullet$ uncaging

[1] P. D. Ray, B.-W. Huang, Y. Tsuji, Cell. Signal. 2012, 24, 981-990.

[2] D. P. Jones, Methods Enzymol. 2002, 348, 93-112.

[3] M. Wang, R. J. Kaufman, Nature 2016, 529, 326-335.

[4] H. Zhang, P. Limphong, J. Pieper, Q. Liu, C. K. Rodesch, E. Christians, I. J. Benjamin, FASEB J. 2012, 26, $1442-1451$. 
[5] A. Trivedi, P. S. Mavi, D. Bhatt, A. Kumar, Nat. Commun. 2016, 7, 11392.

[6] P. Klán, T. Šolomek, C. G. Bochet, A. Blanc, R. Givens, M. Rubina, V. Popik, A. Kostikov, J. Wirz, Chem. Rev. 2013, 113, 119-191.

[7] C. Brieke, F. Rohrbach, A. Gottschalk, G. Mayer, A. Heckel, Angew. Chem. Int. Ed. 2012, 51, 8446-8476; Angew. Chem. 2012, 124, 8572-8604.

[8] G. C. R. Ellis-Davies, Chem. Rev. 2008, 108, 1603-1613.

[9] C. Gwizdala, S. C. Burdette, Curr. Op. Chem. Biol. 2013, 17, 137-142.

[10] R. H. Kramer, D. L. Fortin, D. Trauner, Curr. Op. Neurobiol. 2009, 19, 544-552.

[11] D. Hoeglinger, A. Nadler, C. Schultz, Biochim. Biophys. Acta Mol. Cell Biol. Lipids 2014, 1841, 1085-1096.

[12] Q. Liu, A. Deiters, Acc. Chem. Res. 2014, 47, 45-55.

[13] D. S. Lawrence, Curr. Op. Chem. Biol. 2005, 9, 570-575.

[14] A. S. Baker, A. Deiters, ACS Chem. Biol. 2014, 9, 1398-1407.

[15] J. A. Burns, J. C. Butler, J. Moran, G. M. Whitesides, J. Org. Chem. 1991, 56, 2648-2650.

[16] P. Hu, T. Feng, C.-C. Yeung, C.-K. Koo, K.-C. Lau, M. H. W. Lam, Chem. Eur. J. 2016, 22, 11537-11542.

[17] L. Shah, S. T. Laughlin, I. S. Carrico, J. Am. Chem. Soc. 2016, 138, 5186-5189.

[18] J. C. Vaughan, G. T. Dempsey, E. Sun, X. Zhuang, J. Am. Chem. Soc. 2013, 135, 1197-1200.

[19] L. Yuan, W. Lin, J. Song, Chem. Commun. 2010, 46, 7930-7932.

[20] A. Alouane, R. Labruère, T. Le Saux, F. Schmidt, L. Jullien, Angew. Chem. Int. Ed. 2015, 54, 7492-7509; Angew. Chem. 2015, 127, 7600-7619.

[21] A. Cossarizza, R. Ferraresi, L. Troiano, E. Roat, L. Gibellini, L. Bertoncelli, M. Nasi, M. Pinti, Nat. Protoc. 2009, 4, 17901797.

[22] D. E. Graham, K. C. Harich, R. H. White, Anal. Biochem. 2003, 318, 325-328.

[23] E. S. Christians, I. J. Benjamin, Am. J. Physiol. Hear. Circ. Physiol. 2012, 302, H24-H37.

[24] D. R. Beriault, G. H. Werstuck, Biochim. Biophys. Acta Mol. Cell Res. 2013, 1833, 2293-2301.

[25] S. Berlin, E. C. Carroll, Z. L. Newman, H. O. Okada, C. M. Quinn, B. Kallman, N. C. Rockwell, S. S. Martin, J. C. Lagarias, E. Y. Isacoff, Nat. Methods 2015, 12, 852-858.

[26] D. Axelrod, D. E. Koppel, J. Schlessinger, E. Elson, W. W. Webb, Biophys. J. 1976, 16, 1055-1069. 